\title{
Editorial: Mobile Recommendations for Location-Based Services and Social Networks
}

\author{
Honghao Gao ${ }^{1}$ Y Yuyu Yin ${ }^{2}$ \\ Published online: 30 October 2020 \\ (C) Springer Science+Business Media, LLC, part of Springer Nature 2020
}

\section{Editorial comment}

In recent years, more and more geo-labelled data are available benefit from advanced hardware (positioning systems, environmental sensors), software (standards, tools, network services) and the ever-growing mentality of sharing (crowdsourcing for geographic tagging). Based on human activities, many daily web/App services (Facebook, Tweeter, and Foursquare) generate data and traces that are often transparently annotated with location and contextual information. And such services make it easier to collect and combine rich and diverse information about locations. Exploiting geo-labelled data provides a tremendous potential to materially improve existing and offer novel types of recommendation services. Those recommendation services bring benefits for many domains, including social networks, marketing and tourism. This special issue includes five selected papers with high quality.

The first article titled "Contactless Continuous Activity Recognition based on Meta-Action Temporal Correlation in Mobile Environments "focuses on three key problems

Honghao Gao

gaohonghao@shu.edu.cn

Yuyu Yin

yinyuyu@hdu.edu.cn

1 School of Computer Engineering and Science, Shanghai University, No 99 of Shangda Road, Shanghai 2004444, China

2 College of Computer Science and Technology, Hangzhou Dianzi University, 155 Wenyi Rd, Wensan Road Electron InformationBlock, Hangzhou 310018, China

in RF-based CAR: denoising, segmentation and recognition. This paper presents the design and implementation of a contactless and sensorless continuous activity recognition system, namely WiCheck, which utilizes the temporal correlation between two adjacent actions in continuous activity to eliminate the cumulative error in continuous activity segmentation. The second article is "RFnet: Automatic Gesture Recognition and Human Identification using Time Series RFID Signals", which proposes RFnet, a multi-branch 1D-CNN based framework. RFnet explores the possibility of directly utilizing time series RFID signal to recognize static/dynamic gestures as well as the identity of users. RFnet can benefit a large number of applications such as smart homes where security is a prior concern. The third article titled "Three-tier Architecture Supporting QoS Multimedia Routing in Cloud-assisted MANET with $5 G$ Communication (TCM5G)" came up with a scheme, where partitioning and clustering are performed to optimize the cluster size. Specifically, partitioning is performed by the improved monarch butterfly optimization algorithm and clustering is selected by computing the importance rate. The fourth paper is "P2P Network Based Smart Parking System Using Edge Computing", which studies a friendly and effective smart parking system in a large city. This paper proposes a $\mathrm{P} 2 \mathrm{P}$ network based smart parking system using Edge Computing. The fifth article is "Service Function Chain Placement for Joint Cost and Latency Optimization" formulates a multi-objective optimization model to joint VNF placement and link embedding in order to reduce deployment cost and service latency with respect to a variety of constraints. And then the paper solves the optimization problem using two heuristic-based algorithms that perform close to optimum for large scale cloud/edge environments. 
Acknowledgments The guest editors are thankful to our reviewers for their effort in reviewing the manuscripts. We also thank the Edit-inChief, Dr. Imrich Chlamtac, for his supportive guidance during the entire process.

Publisher's note Springer Nature remains neutral with regard to jurisdictional claims in published maps and institutional affiliations.

Honghao Gao received the Ph.D. degree in Computer Science and started his academic career at Shanghai University in 2012. Prof. Gao is currently with the School of Computer Engineering and Science, Shanghai University, China. He is also a Professor at Gachon University, South Korea. Prior to that, he was a Research Fellow with the Software Engineering Information Technology Institute of Central Michigan University (CMU), USA, and was also an Adjunct Professor at Hangzhou Dianzi University, China. His research interests include service computing, model checking-based software verification, wireless networks, and intelligent medical image processing. He has publications in IEEE TII, IEEE T-ITS, IEEE IoT-J, IEEE TNSE, IEEE TCCN, IEEE/ ACM TCBB, ACM TOIT, ACM TOMM, IEEE TCSS, IEEE TETCI, IEEE Network, and IEEE JBHI. Prof. Gao is a Fellow of IET, BCS, and EAI, and a Senior Member of IEEE, CCF, and CAAI. He is the Editor-inChief for International Journal of Intelligent Internet of Things Computing (IJIITC), and Associate Editor for IET Software, Wireless Network, International Journal of Communication Systems, IET
Wireless Sensor Systems, IET The Journal of Engineering, and Journal of Medical Imaging and Health Information. Moreover, he has broad working experiences in industry-university-research cooperation. He is a European Union Institutions appoint external expert for reviewing and monitoring EU Project, is a member of the EPSRC Peer Review Associate College for UK Research and Innovation in the UK, and is also a founding member of IEEE Computer Society Smart Manufacturing Standards Committee.

Yuyu Yin received the Ph.D. degree in computer science from Zhejiang University, Hangzhou, China, in 2010. He is currently a Professor with the College of Computer Science and Technology, Hangzhou Dianzi University, Hangzhou, China. During the past ten years, he has authored or coauthored more than 40 papers in journals and has served as a referee in conferences, such as Sensors, Entropy, International Journal of Software Engineering and Knowledge Engineering (IJSEKE), Mobile Information Systems, International Conference on Web Services, and Software Engineering and Knowledge Engineering. His research interests include service computing, cloud computing, and business process management. He is a member of the CCF and a CCF Service Computing Technical Committee member. He has organized more than ten international conferences and workshops, such as Formal Methods in Services and Cloud Computing from 2011 to 2017 and Digital Intelligence for Systems and Machines from 2012 to 2018. Additionally, he worked as a Guest Editor for the Journal of Information Science and Engineering and IJSEKE and as a Reviewer for the IEEE TRANSACTION ON INDUSTRIAL INFORMATICS, Journal of Database Management, Future Generation Computer Systems, etc. 